\title{
3 Die Waffen-SS im Krieg
}

Seit Kriegsbeginn im September 1939 agierten bewaffnete SS-Einheiten gemeinsam mit Sicherheitspolizei und Sicherheitsdienst (SD) in den rückwärtigen Gebieten und zeichneten für zahlreiche Kriegsverbrechen verantwortlich. Die auf verschiedene Heeresteile verteilten SS-Einheiten sollten nun zusammengefasst und im November 1939 in Waffen-SS umbenannt werden. ${ }^{1}$ Im Oktober 1939 begann die Aufstellung der SS-Verfügungs-Division (der späteren Division „Das Reich“). Zugleich wurde aus den SS-Totenkopfstandarten „Oberbayern“, „Brandenburg“ und „Thüringen“, der SS-Heimwehr „Danzig“ sowie dem Großteil der KZ-Wachmannschaften die SS-Totenkopf-Division gebildet. Weiteres Personal für diesen neuen Großverband kam von der Allgemeinen SS und der SS-Verfügungstruppe. Aus Angehörigen der Ordnungspolizei und einigen Einheiten der Wehrmacht erfolgte schließlich noch die Aufstellung der SS-Polizei-Division. ${ }^{2}$

Die Eroberungskriege eröffneten der bewaffneten SS bzw. Waffen-SS seit dem Überfall auf Polen 1939 zwar neue Betätigungsfelder, gleichzeitig verlor ihr Ordenscharakter mit den drei Grundpfeilern „Freiwilligkeit“, „rassische Auslese“ und „weltanschauliche Erziehung“ im Verlauf des Krieges an Bedeutung und wurde durch das starke Wachstum und die hohen Verluste seit Beginn des Ostfeldzugs im Juni 1941 zunehmend in Frage gestellt. ${ }^{3}$

Die Organisation „Waffen-SS“ befand sich aufgrund dieser Entwicklung in einem permanenten inneren als auch äußeren Spannungsverhältnis: Das innere war geprägt durch das Auseinanderklaffen von idealer Selbstwahrnehmung und realem Ist-Zustand, also der Diskrepanz zwischen dem Ideal einer Elite und der Realität einer Organisation, die spätestens seit 1942 so gut wie jeden aufnahm. ${ }^{4}$ Das äußere Spannungsverhältnis war gekennzeichnet durch zunehmende Rivalitäten zwischen Waffen-SS und Wehrmacht sowie zwischen ersterer und anderen Institutionen des Dritten Reiches. Der Zugriff auf personelle Ressourcen, der bis 1939 vor allem durch die Wehrmacht eingeschränkt war, wurde im Zuge eroberter oder unter deutschem Einfluss stehender Länder zwar erleichtert, doch die

1 Vgl. Leleu, La Waffen-SS, S. 23. Vgl. Wegner, Waffen-SS, S. $126 \mathrm{f}$.

2 Vgl. Mehner, Waffen-SS und Polizei, Bd 3. Noderstedt: Militair-Verlag Klaus D. Patzwall 1995, S. 11. Vgl. Leleu, La Waffen-SS, S. 21.

3 Vgl. Wegner, Soldaten, S. 264. Leleu, Jenseits der Grenzen, S. 35, 37. Vor dem Krieg hatten nicht einmal 10\% der SS-Angehörigen einer bewaffneten Einheit angehört. Vgl. Wegner, Soldaten, S. 264.

4 Vgl. hierzu Kapitel zur „Prinz Eugen“.

Ә Open Access. (c) 2021 Franziska Anna Zaugg, publiziert von De Gruyter. (c) BY lizenziert unter einer Creative Commons Namensnennung 4.0 International Lizenz. https://doi.org/10.1515/9783110730777-003 
Konkurrenz zur Wehrmacht war damit nicht behoben, denn auch sie erhob Anspruch auf potentielle ausländische Kontingente zur Entlastung der eigenen Verbände. ${ }^{5}$ Die daraus resultierenden Auseinandersetzungen lassen sich beispielhaft in den Gebieten des NDH wie auch für Albanien, Kosovo und die Sandžak-Region nachweisen. ${ }^{6}$

\subsection{Von der „Schutzstaffel“ zum multinationalen Massenheer}

Die seit 1936 durch die Allgemeine SS zu erfüllenden Werbequoten für die bewaffneten Verbände konnten trotz Anreizen, wie beispielsweise einer sicheren beruflichen Zukunft, nicht eingehalten werden und führten im Dezember 1938 zur Lockerung der Zulassungsbestimmungen. ${ }^{7}$ Als auch diese Maßnahme nicht ausreichte, um die geforderten Rekrutierungskontingente einzuhalten, traf das für die Rekrutierungen der bewaffneten SS zuständige SS-Ergänzungsamt, dessen Chef Gottlob Berger war, die Entscheidung, neue Gebiete für die Rekrutierung zu erschließen: Seit Ende 1938 bzw. Anfang 1939 durften sich auch Deutsche aus den tschechischen Sudetengebieten für die SS-Totenkopfverbände melden. ${ }^{8}$

In den Jahren nach 1940 wuchs die Waffen-SS rasch an und die einzige Möglichkeit, neu aufgestellte Divisionen aufzufüllen und Verluste zu ersetzen, bestand darin, „Hilfsvölker“ heranzuziehen. Dies aber stand im Gegensatz zu den rassischen „Ausleseprinzipien“ der SS. ${ }^{9} \mathrm{Zu}$ dieser Notwendigkeit, die nicht nur die Waffen-SS, sondern auch die Wehrmacht betraf, äußerte sich der Oberbefehlhaber Südost, Generalfeldmarschall Maximilian von Weichs, der die Heeresgruppe F führte, mit folgenden Worten:

Um die Lücken der mit riesigen Frontbreiten eingesetzten Divisionen zu schließen, sind tausende von Wehrunwürdigen eingesetzt, ist auf Fremdstämmige aller Nationalitäten zurückgegriffen. So stehen bis zuletzt arabische, russische und italienische Bataillone Schulter an Schulter neben den deutschen Landesschützen im Sicherungsdienst. Mit unendlicher Mühe werden die Elemente der Ordnung jedes einzelnen Balkanlandes gesammelt, bewaffnet, ausgebildet und miteingesetzt im Kampf gegen den gemeinsamen kommunistischen Feind.

5 Siehe zur Vielfalt und Zusammensetzung „fremdvölkischer“ Waffen-SS Angehörigen: Böhler, Gerwarth, The Waffen-SS, S. 11-13.

6 Vgl. Zaugg, Albanische Muslime, S. 107-113.

7 Vgl. Wegner, Soldaten, S. 137. Vgl. Rohrkamp, Weltanschaulich gefestigte Kämpfer, S. 207.

8 Vgl. Rohrkamp, Weltanschaulich gefestigte Kämpfer, S. 207.

9 Vgl. Birn, SS-Ideologie und Herrschaftsausübung, S. 61. Leleu hingegen führt an, dass es nicht zur eigentlichen Aufgabe des Elite-Prinzips kam, sondern zu dessen Weiterentwicklung mit verschiedenen Abstufungen. Vgl. Leleu, La Waffen-SS, S. 61-87. 
So wachsen griechische, albanische, [bosnische], montenegrinische und serbische Milizverbände aus dem Boden. ${ }^{10}$

Doch mit dem Fortdauern des Krieges und der Expansion der Waffen-SS zum multikulturellen Massenheer mehrten sich die Vorwürfe, viele dieser Divisionen würden nur aufgestellt, um sie als Kanonenfutter an ausgeblutete Frontabschnitte zu schicken. ${ }^{11}$ Bei Kriegsende sollten Ausländer und „Volksdeutsche“ schließlich die große Mehrheit der Organisation stellen, die sich einst als rassische Elite des Nationalsozialismus präsentierte. ${ }^{12}$ Doch einer von mehr als 800.000 Waffen-SSSoldaten zu sein und in derselben Uniform zu kämpfen, bedeutete nicht, dieselben Privilegien zu haben. ${ }^{13}$ Die Formationen unterschieden sich bezüglich ihrer Zusammensetzung, Schlagkraft, ihrer Ausbildung und Ausrüstung, ihrem Verhalten gegenüber dem Feind, wie auch bezüglich Ort und Zeitpunkt, wo sie eingesetzt wurden, stark. ${ }^{14}$

Spätestens durch die enormen Verluste an der Ostfront, der verlorenen Schlacht um Moskau im Winter 1941/42 und den weiterhin unklaren Verhältnissen auf dem Balkan setzte in der Rekrutierung von Waffen-SS-Soldaten eine Entwicklung ein, die sich in zweierlei Hinsicht von bisherigen Rahmenbedingungen

10 OB Südost (Heeresgr. F) Generalfeldmarschall Maximilian von Weichs, H.Q., „Die große Absetzbewegung im Südosten“, Januar 1945, BArchF, RH 19-XI/86, S. 2.

11 Ausbildungszeiten, Material, aber auch Personalressourcen unterschieden sich an verschiedenen Fronten bzw. sogar Frontabschnitten aber auch zwischen Wehrmacht und Waffen-SS teilweise stark: Vgl. bspw. Peter Lieb, Militärische Elite? Die Panzerdivisionen von Waffen-SS und Wehrmacht in der Normandie 1944 im Vergleich, in Schulte, Lieb, Wegner, Die Waffen-SS, S. 337-341. Albanische Rekruten mussten zum selben Zeitpunkt mit Beutewaffen, einer zusammengewürfelten Ausrüstung und heruntergekommenen italienischen Unterkünften vorlieb nehmen. Ihre militärische Ausbildung dauerte nur sechs Wochen. Vgl. Zaugg, Albanische Muslime, S. 234-239, 241f. Die in der weiteren Arbeit vorgestellten ausländischen Formationen konnten von der Ausrüstung, die beispielsweise den drei SS-Panzerdivisionen „Leibstandarte“, „Das Reich“ und „Totenkopf“ beim Unternehmen „Zitadelle“ im Sommer 1943 zur Verfügung stand, nur träumen. Siehe hierzu ausführlich Roman Töppel, Waffen-SS und Wehrmacht bei der Schlacht bei Kursk. Ein Vergleich im operativen Einsatz, in: Schulte, Lieb, Wegner, Die Waffen-SS, S. 320-322.

12 Vgl. Casagrande, Volksdeutsche SS-Division, S. 323.

13 Vgl. Leleu, La Waffen-SS, S. 87.

14 Vgl. hier auch Peter Liebs Untersuchung, Militärische Elite?, S. 336-253. Lieb kommt dabei zum Schluss, dass sich das Verhalten von Soldaten der Wehrmacht bzw. der Waffen-SS insbesondere in Bezug auf Kriegsverbrechen deutlich unterschied. Vg. ebd., S. 353. Hierzu wird die in Entstehung begriffene Disseration von Chris Helmecke, Waffen-SS im Fronteinsatz. Kriegführung und Kampfwert der SS-Panzerdivisionen 1939-1945 am Zentrum für Militärgeschichte und Sozialwissenschaften der Bundeswehr in Potsdam weitere erhellende Einblicke liefern. 
unterschied: Erstens sollten im Frühjahr 1942 zum ersten Mal massenhaft "Volksdeutsche", insbesondere aus Südosteuropa, geworben werden, und zweitens geschah dies nachweislich häufig auf einer nicht mehr freiwilligen Basis, wie etwa Sundhaussen festhält: „Hatte sie [die Waffen-SS] vorher den Charakter einer Elitetruppe, die überwiegend deutsch war und im wesentlichen aus Freiwilligen bestand, so begann sich in der zweiten Hälfte $1942 \mathrm{ihr}$ Gesicht zu wandeln. “15 Er bezog sich dabei auf Stein, der seinerseits betonte, dass durch diese Ausweitung und Veränderung der Rekrutierungstaktik, sowohl das Ziel der „ethnischen Einheitlichkeit“, indem auch „nicht-germanische“ Rekruten aufgenommen wurden, als auch dasjenige, dass die SS „eine Elitetruppe aus Freiwilligen“ sei, endgültig über Bord geworfen wurden. ${ }^{16}$

Seit der Niederlage von Stalingrad im Winter 1942/43, die bis heute als Wendepunkt des Zweiten Weltkriegs bezeichnet wird, ${ }^{17}$ und dem damit einhergehenden raschen Anwachsen der Partisanenarmeen in Ost- und Südosteuropa bedurfte es aufgrund der zunehmend prekären Personalsituation noch mehr Neurekrutierungen; einerseits, um bestehende Divisionen aufzufüllen und andererseits, um zusätzliche neue Divisionen aufzustellen. ${ }^{18}$ Es ist daher leicht nachvollziehbar, dass nach dieser schweren militärischen Niederlage und den extremen Verlusten an der Ostfront auch die massenhafte Rekrutierung von „Fremvölkischen“ in die Waffen-SS einsetzte: ${ }^{19}$ „Nur elf Tage nach der Aufgabe der letzten deutschen Verteidigungsstellungen an der Wolga“, so Leleu, „stand Hitler nicht nur dem Vorschlag zur Schaffung einer aus der Hitlerjugend zu rekrutierenden Division positiv gegenüber, sondern erteilte Himmler auch die Erlaubnis, eine lettische, eine litauische und eine bosnische Division aufzustellen.“20

15 Sundhaussen, Waffen-SS in Kroatien, S. 189. Gerhard Rempel, Gottlob Berger: ,Ein Schwabengeneral der Tat', in: Ronald Smelser und Enrico Syring (Hgg.), Die SS: Elite unter dem Totenkopf. 30 Lebensläufe, Paderborn: Schöningh 2000, S. 50.

16 Sundhaussen, Waffen-SS in Kroatien, S. 189. Stein, Geschichte der Waffen-SS, S. 154.

17 Zur Differenzierung dieser Schlacht als Wendepunkt innerhalb des Zweiten Weltkriegs weist Wegner aber darauf hin, dass „von Stalingrad als einer Kriegswende ganz allgemein und uneingeschränkt zu sprechen“, das heisst, ohne auf die Vielschichtigkeit der deutschen wie auch der alliierten Kriegführung einzugehen, auf ihre unterschiedlichen Ausgangslagen, Ziele, Ressourcen etc. irreführend sei. Bernd Wegner, Grundprobleme der deutschen Kriegführung nach Stalingrad, in: Karl-Heinz Frieser u. a. (Hgg.): Die Ostfront 1943/44. Der Krieg im Osten und an den Nebenfronten (Das Deutsche Reich und der Zweite Weltkrieg, Bd. 8). München: Deutsche Verlags-Anstalt 2007, S. 7.

18 Leleu, La Waffen-SS, S. 547-549.

19 Vgl. Casagrande, Volksdeutsche SS-Division, S. 325. Vgl. Sundhaussen, Waffen-SS in Kroatien, S. 182. Bernd Wegner, Grundprobleme der deutschen Kriegführung nach Stalingrad, S. 8-11.

20 Leleu, Jenseits der GrenzenS. 27. 
Entsprechend fällt die Aufstellung von drei Viertel der Waffen-SS-Divisionen in die Zeit nach Stalingrad, zwischen Februar 1943 bis Mai 1945. Auch die Ausweitung der Rekrutierungen auf die muslimische Bevölkerung Südosteuropa 1943 ist vor diesem Hintegrund zu sehen. Leleu relativiert aber, dass der Gedanke einer eigentlichen Elite nie aufgegeben worden sei, denn durch Vermerke in den Stammbüchern hätte nach einem gewonnenen Krieg einwandfrei festgestellt werden können, wer zum „inneren Kreis“ der Waffen-SS zählte und wer nicht. ${ }^{21}$ Deutsche bzw. österreichische SS-Angehörige selbst betrachteten südosteuropäische SS-Divisionen als minderwertige Formationen. Eine Versetzung von einer in anderen Teilen Europas gelegenen Division nach Südosteuropa kam einer Strafversetzung gleich. ${ }^{22}$

\subsection{Dreistufenmodell einer paneuropäischen Armee}

Das von Himmler seit Kriegsbeginn vorangetriebene exponentielle Wachstum der SS verlangte nach Möglichkeiten, neue Rekrutierungspools zu erschließen: Himmlers Vision einer paneuropäischen Armee, ${ }^{23}$ zielte nebst dem angestrebten Machtausbau darauf ab, dem Problem steigender Personalressourcen zu begegnen, ohne in Konflikt mit der Wehrmacht zu geraten. Um seine Vision in die Ideologie der SS einzupassen, entwickelte Himmler bereits 1940 ein erstes Dreistufenmodell, welches Waffen-SS-Soldaten in „ordensfähige“ SS-taugliche Deutsche, nicht „ordensfähige“ Deutsche und „Germanen“ einteilte. Davon ausgehend ergab sich folgender Aufbau: Den innersten Kern der SS bildeten die „reichsdeutschen“ Soldaten. Darum herum sollten sich, so die Umsetzungsidee für eine pangermanische Armee, „germanisch“-stämmige, „artverwandte Völker“ und „volksdeutsche“ Rekruten scharen. Im äußersten Kreis befanden sich schließlich die „Fremdvölkischen“, die jedoch erst später in diesen Überlegungen auftauchten. ${ }^{24}$ Somit muss der geplante Ausbau der Waffen-SS zur pangermanischen und später paneuropäischen Armee - als eine gewollte, überlegte und gut inszenierte Strategie und nicht nur als eine Folge der hohen Verluste im Osten

21 Vgl. Leleu, Jenseits der Grenzen, S. 37. Er zeigt am Beispiel des Zeitraumes vom 1. September 1939 bis 31. Dezember 1943, dass die Waffen-SS zwar von 23.000 Mann auf 501.000 Mann anwuchs, davon aber nur 68.000 die Möglichkeit erhielten, dem „Orden“ der SS beizutreten. Vgl. ebd.

22 Vgl. Kumm, Vorwärts Prinz Eugen, S. 39.

23 Vgl. Wegner, Soldaten, S. 310-316.

24 Vgl. Leleu, La Waffen-SS, S. 61. 
gesehen werden. ${ }^{25}$ Auch in der SS-Propaganda wurde seit 1942 entsprechend „der Gedanke eines Europas mit gemeinsamer Geschichte und gemeinsamen Interessen forciert”. ${ }^{26}$ Doch jener Übergang „vom Stoßtrupp der Bewegung zur Kampftruppe für Europa“ und seine Umsetzung war von Schwierigkeiten geprägt: Denn erstens mussten ideologische Bedenken überwunden werden und zweitens wies die Erweiterung auch in der Praxis zahlreiche heikle Punkte auf, angefangen bei einer adäquaten Bezeichnung neuer, ausländischer Verbände bis hin zum alltäglichen Umgang mit ihren Soldaten. ${ }^{27}$

\subsubsection{Die Rekrutierung „germanischer“ Freiwilliger}

Nach der Ausweitung des Krieges auf Westeuropa dehnten Himmler und Berger die Werbung auf nicht-deutsche „Germanen und artverwandte Völker“ aus. Nachdem deutsche Truppen 1940 in Dänemark, Norwegen, den Niederlanden, Belgien und Frankreich einmarschiert waren, schickten sie sich an, den Beitritt zur Waffen-SS auch für „germanische“ Freiwillige dieser Länder zu öffnen. Zu den ersten solcher Einheiten zählten die Regimenter „Nordland“ und „Westland“. ${ }^{28}$ Daraus enstanden die 5. SS-Division „Wiking“ wie auch diverse west- und nordeuropäische Legionen. ${ }^{29}$

25 Vgl. Wegner, Waffen-SS, S. 311. Vgl. hierzu auch Birn, welche die Meinung vertritt, dass der Ausbau nur aufgrund von Sachzwängen erfolgte und weder geplant noch gewünscht war. Birn, SS-Ideologie und Herrschaftsausübung, S. 74.

26 Vgl. Birn, SS-Ideologie und Herrschaftsausübung, S. 65. Vgl. hierzu auch Tim Kirk, Nazi plans for a European order and European responses, in: Johannes Dafinger, Dieter Pohl, A new Nationalist Europe under Hitler. Concepts of Europe. Concepts of Europe and transnational networks in the national socialist sphere of influence, 1933-1945, London etc.: Routledge 2019, S. 71-92.

27 Vgl. Leleu, Jenseits der Grenzen, S. 35f. Hierzu wurden spezielle Anleitungen verfasst, bspw. von Kaesdorf, „Erfahrungen mit albanischen Soldaten“, undatiert, BArchB, NS 31/ 444, S. 28. Vgl. auch „Politische Richtlinien für die Turkverbände und die Ostmuselmanische SS-Division“, 12.2.1944, BArchB, NS31/42 oder „Die politische Aufgabe des deutschen Führers und Unterführers in den nichtdeutschen Einheiten der Waffen-SS“, 20.10.1944, BArchB, NS31/42. Speziell zur Namensgebung siehe auch: Vgl. Leleu, La Waffen SS, S. $73 \mathrm{f}$. 28 Zusammen mit dem Regiment „Germania“ bildeten sie schließlich die 5. SS-Division „Wiking“. Vgl, Claus Bundgard Christensen, Niels Bo Poulsen, Peter Scharff Smith, Germanic volunteers from northern Europe, in: Böhler, Gerwarth, The Waffen-SS, S. $51 \mathrm{f}$.

29 Vgl. Bundgard Christensen, Bo Poulsen, Scharff Smith, Germanic volunteers from northern Europe, in: Böhler; Gerwarth, The Waffen-SS, S. 52. Vgl. auch Sørlie, Sonnenrad und Hakenkreuz. Norweger in der Waffen-SS 1941-45, Paderborn: Ferdinand Schöningh 2019, S. 60-62. 
Die europäische Ausrichtung der Waffen-SS war Teil der Strategie Himmlers und Bergers, die Konkurrenz um Personalressourcen mit der Wehrmacht zu umgehen. ${ }^{30}$ Schon im August 1940 rechnete Berger Himmler vor, welche Kontingente an neuen Waffen-SS-Soldaten wo rekrutiert werden könnten. Im Reich selbst, so vermutete er, könnten mit sinkender Tendenz 12.000 pro Jahr geworben werden, in Frankreich, Belgien und den Niederlanden bei einer zweiprozentigen Auslese 2.664 Rekruten pro Jahr. Mit deutlich weniger, nämlich 1.234 Neuzugängen pro Jahr, rechnete er bei gleichen Bedingungen für Dänemark, Norwegen und Schweden. In seinen Überlegungen spielten auch mögliche Rekruten aus Übersee eine wesentliche Rolle: Er liebäugelte mit 5,5 Millionen deutschstämmigen Nordamerikanern und Kanadiern, 1,2 Millionen ebensolchen in Südamerika und 77.000 in Australien. ${ }^{31}$ Wegner sieht in diesem von „Berger vorgeschlagene[n] Ausweichen auf einen anderen, außerhalb der deutschen Reichsgrenzen gelegenen ,Markt‘ [...] nicht zuletzt die ideologisch konsequente Reaktion auf ein praktisches Problem.“32

Ein wesentlicher Schritt in der geographischen und quantitativen Ausdehnung von Rekrutierungen stellte die „Germanischen Leitstelle“ dar, welche seit 1941 alle Aktivitäten koordinierte, die die „germanische“ Freiwilligenwerbung betrafen. $^{33}$

\subsubsection{Die Rekrutierung „Volksdeutscher“}

In kleineren Gruppen und in unsystematischer Weise waren bereits vor Kriegsbeginn „Volksdeutsche“ rekrutiert worden. ${ }^{34}$ Bereits seit Ende des Ersten Weltkriegs gewannen diese ethnischen Deutschen außerhalb der Grenzen des Reiches an politischer Bedeutung. Die „Volksdeutschen“ wurden vermehrt als Teil der deutschen Gemeinschaft wahrgenommen und stilisiert, obwohl einige der von ihnen bewohnten Gebiete nie zum Deutschen Reich gehört hatten, andere hingegen nach der Niederlage im letzten Krieg anFrankreich oder Polen abgetreten werden mussten. ${ }^{35}$ Schon seit Kriegsbeginn 1939 und dem durch deutsche Erfolge erzielten Gebietszuwachs begann sich Berger, für die „volksdeutschen“

30 Vgl. beispielsweise Leleu, Jenseits der Grenzen, S. 39.

31 Vgl. Wegner, Soldaten, S. 311.

32 Ebd., S. 312.

33 Vgl. Harten, Himmlers Lehrer, S. 355. Ausführlich zur „Germanischen Leitstelle“ siehe ebd., S. $355-368$.

34 Vgl. Wegner, Soldaten, S. 310. Böhler; Gerwarth, The Waffen-SS, S. $10 \mathrm{f}$.

$35 \mathrm{Vgl}$. Casagrande/Schvarc/Spannenberger/Traşca, Volksdeutsche, S. 209. 
Gemeinschaften Europas und damit zusammenhängende Personalressourcen zu interessieren. So halfen beispielsweise die unter dem Kommando der SS in "Volksdeutsche Selbstschutz"-Einheiten konzentrierten deutschstämmigen Polen mit, in den ersten Monaten des Zweiten Weltkriegs zehntausende PolInnen und JüdInnen zu ermorden. ${ }^{36}$ Bergers Aufmerksamkeit richtete sich aber schon bald insbesondere auf die „volksdeutschen“ Gemeinschaften Südosteuropas. Mit der Rekrutierung „Volksdeutscher“ hoffte er nicht zuletzt, „reichsdeutsche“ Kräfte zu schonen und Auseinandersetzungen mit der Wehrmacht um Personalressourcen zu vermeiden. ${ }^{37}$

Die massenhafte Integration bzw. gezielte Rekrutierung „Volksdeutscher“ aus den betreffenden europäischen Regionen, vor allem aus Rumänien, Ungarn und Serbien, aber auch aus Kroatien und Tschechien sollte in der Ausdehnung der Waffen-SS einen weiteren großen Schritt darstellen. Doch auch in anderen Staaten wie Polen, Italien (Südtirol), Frankreich (Elsass) oder Belgien waren „volksdeutsche“ Gemeinschaften zu finden, die für den Dienst in der Waffen-SS geworben werden konnten. ${ }^{38}$

Bereits 1940 waren rund 20 Prozent der Waffen-SS-Mitglieder sogenannte „Volksdeutsche“; in den Folgejahren stieg der Anteil „Volksdeutscher“ in der Waffen-SS auf rund 33 Prozent. „Volksdeutsche“, die aus dem „Altreich“ bzw. der „Ostmark“ oder auch dem Südtirol stammten, konnten durch ihre Einbürgerung frei entscheiden, ob sie der Wehrmacht oder der Waffen-SS beitreten wollten. Diese Wahlmöglichkeit hatten „Volksdeutsche“ in Südosteuropa meist nicht. ${ }^{39}$

Im Sommer 1941 erließ das Reichsinnenministerium aufgrund der neu eroberten Gebiete im Osten und Südosten eine Regelung, welche Bevölkerungsgruppen als „Volksdeutsche“ zu betrachten seien: „Volksdeutsche“ waren „diejenigen Personen nicht deutscher Staatsangehörigkeit, die sich zum deutschen Volkstum bekennen, sofern dieses Bekenntnis durch bestimmte Tatsachen (Sprache, Erziehung, Kultur, Abstammung usw.) gestützt wird.“40 Ähnlich wie später bei den „Muslimen“ verfolgte die NS-Propaganda das Ziel, diese unterschiedlichen Gruppen unter dem Label „Volksdeutssche“ als homogene Masse erscheinen zu lassen. Bei näherer Betrachtung jener über hunderttausend südosteuropäischen „Volksdeutschen“, die schließlich in der Waffen-SS kämpften, springt jedoch so-

36 Vgl. Böhler; Gerwarth, The Waffen-SS, S. 10.

37 Vgl. Sundhaussen, Waffen-SS in Kroatien, S. 177.

38 Vgl. Böhler; Gerwarth, The Waffen-SS, S. $10 \mathrm{f}$.

39 Vgl. Casagrande, Südtiroler in der Waffen-SS, S. 34.

40 OKW/Amt Ausl/Abw/Abt.Abw.III Nr. 2123/5.41g. (IIIC5) vom 15.7.1941 betr. Behandlung von Volksdeutschen, BArchF, RW 4/V.300 zitiert nach Hans Umbreit, Auf dem Weg zur Kontinentalherrschaft, in: Kroener et al., Organisation und Mobilisierung, S. 266. 
fort ihre ethnische Heterogenität ins Auge. Schließlich handelte es sich zum Beispiel bei der „Prinz Eugen“ mitnichten um einen homogenen Verband, wie der Name suggerierte, sondern um eine ethnisch stark durchmischte WaffenSS-Division. ${ }^{41}$

Gegen Ende des Zweiten Weltkriegs dienten schließlich rund 150.000 „Volksdeutsche“, ein Großteil davon aus Südosteuropa, in der Waffen SS. ${ }^{42}$ Heinz Höhne errechnete sogar ein Total von über 300.000 „Volksdeutschen“ aus allen Teilen Europas, was aus heutiger Sicht $\mathrm{zu}$ hoch gegriffen scheint. ${ }^{43}$ Lange vor Ende des Zweiten Weltkriegs übertraf aber die Anzahl der im Ausland geborenen die in Deutschland zur Welt gekommenen Waffen-SS Soldaten. ${ }^{44}$

\subsubsection{Die Rekrutierung „Fremdvölkischer“}

Die Ausdehnung zu einer "pangermanischen" und schließlich einer "paneuropäischen” Armee war nicht nur Sachzwängen geschuldet, sondern auch Teil von Himmlers Wachstumsstrategie. ${ }^{45}$ Auf sein Drängen, die Waffen-SS möge an Einfluß gewinnen, aber auch aufgrund der Kriegsnotwendigkeit, wurde die Waffen-SS 1943 auch für „Nicht-Germanen“ aus Ost- und Südosteuropa geöffnet. Es entstanden unter anderen die 13. kroatische, die 14. galizische, die 15. lettische, etwas später die 19. lettische, die 20. estnische und die 21. albanische Freiwilligen-Division. Daneben wurden Freiwillige für zwei russische, eine „weissruthenische“ und drei ungarische Divisionen geworben. ${ }^{46}$ Allerdings handelte es sich bei diesen Divisionen vielfach nur noch dem Namen nach um „Freiwilligen“-Formationen und um „Divisionen“. Nur wenige Nichtdeutsche schlossen sich der Waffen-SS noch aus ideologischer Überzeugung an. Konnte man „Volksdeutsche“ noch mit Verweis auf germanischen Gemeinsamkeiten überzeugen, so

41 Vgl. hierzu beispielsweise die Aussage des ehemaligen Divisionsangehörigen Günter Borkowsky, USHMM, BArch Ludwigsburg B162/20812, Bl. 6.

42 Vgl. Casagrande/Schvarc/Spannenberger/Traşca, Volksdeutsche, S. 209.

43 Vgl. Höhne, Orden unter dem Totenkopf, S. 425.

44 Vgl. Sundhaussen, Waffen-SS in Kroatien, S. 178.

45 Vgl. Wegner, Waffen-SS, S. 311. Über einzelne „fremdvölkische“ Verbände und Einheiten der Waffen-SS wird indes schon länger geforscht. Für den südosteuropäischen Raum sind etwa Broszats 1966 erschienenes Gutachten für das Institut für Zeitgeschichte zu albanischen Milizen im Zweiten Weltkrieg oder Sundhaussens Aufsatz zur Geschichte der Waffen-SS in Kroatien von $1971 \mathrm{zu}$ nennen. Vgl. Martin Broszat, Albanische Milizen im Zweiten Weltkrieg, in: Gutachten des Instituts für Zeitgeschichte 2 (1966), S. 343-347. Vgl. Sundhaussen, Holm: Zur Geschichte der Waffen-SS in Kroatien 1941-1945, in: Südostforschungen 30 (1971), S. 176-196.

46 Vgl. Harten, Himmlers Lehrer, S. 409. 
gestaltete sich die Überzeugungsarbeit bei „nicht-germanischen“ bzw. „fremdvölkischen" Soldaten schwieriger. Wie wir sehen werden, reichten die Faktoren, die sie in die Waffen-SS eintreten ließen, von einer prekären wirtschaftlichen Situation, über die Bedrohung durch andere militärische Gruppierungen, zu unmenschlichen Haftbedingungen in deutschen Kriegsgefangenenlagern bis hin zu politischen Zielen für das Nachkriegseuropa. ${ }^{47}$

Knapp zwei Wochen nach der Kapitulation der deutschen 6. Armee im Kessel von Stalingrad, am 13. Februar 1943, gab Hitler grünes Licht für die Aufstellung der ersten „fremdvölkischen“ Divisionen: einer kroatischen SS-Freiwilligen-Division, bestehend aus muslimischen Bosniern und katholischen Kroaten und schließlich 13. Waffen-Gebirgs-Division der SS „Handschar“ genannt, einer galizischen Division, die 14. Waffen-Grenadier-Division der SS, bestehend aus Ukrainern und „Volksdeutschen“ und einer lettischen, die 15. Waffen-Grenadier-Division der SS. ${ }^{48}$ Die Abstufung von oben genannten „ordensfähigen“ zu diesen „nicht ordensfähigen“ Verbänden zeigte sich auch in ihrer Namensgebung, bei welcher das Kürzel „SS“ am Schluss genannt wird. Neue bewaffnete SS-Verbände entstanden nun in rascher Folge: Schon ein Jahr später, am 13. April 1944, verkündete der Chef des SS-Führungshauptamtes, SS-Obergruppenführer Hans Jüttner auf der SS-Führer-Tagung in Prag, dass Himmler die Aufstellung von insgesamt 53 Divisionen ins Auge fasse; ${ }^{49}$ bei Kriegsende existierten schließlich 38 Waffen-SS-Divisionen. ${ }^{50}$

In drei dieser „fremdvölkischen“ Divisionen dienten vor allem Muslime. Setfan Petke, der in seiner Dissertation die Rekrutierung von Muslimen in Wehr-

47 Vgl. Harten, Himmlers Lehrer, S. 411. Siehe zur Entlassung aus der Kriegsgefangenschaft und Dienst in der SS auch das Beispiel der „Trawniki“-Männer. Vgl. Sara Berger; Angelika Censebrunn-Benz, Zwischen Deutschen und Juden: Die „Trawnikis“ in den Vernichtungslagern der „Aktion Reihardt“, in: Zaugg; Młynarczyk (Hgg.): Ost- und Südosteuropäer in der WaffenSS, ZfG 7/8 (2017), beispielsweise S. 628. Auf spezifische Interessen und Zwänge der einzelnen Gruppierungen wird in separaten Kapiteln detailliert eingegangen.

48 Vgl. Leleu, Jenseits der Grenzen, S. 27. Vgl. Black, Gutmann, Racial theory, S. 35. Leleu, La Waffen SS, S. 76. Vgl. hierzu auch frühe Rekrutierungen in Estland, Lettland und Litauen bereits während des deutschen Einmarschs 1941, Vgl. Böhler, Gerwarth, The Waffen-SS, S. $11 \mathrm{f}$.

49 Vgl. Rede SS Obergruppenführer Jüttner auf der SS-Führer-Tagung in Prag, 13.4.1944, BArchB NS 33/31, Bl.10-12, zitiert nach: Leleu, Jenseits der Grenzen, S. 28.

50 Leleu geht von über 800.000 Mann aus, die bis Kriegsende in den Reihen der Waffen-SS gedient haben. Vgl. Leleu, La Waffen SS, S. 87. Diese Zahl ist aber zu niedrig. Höhne nennt 910.000 Mann, Vgl. Höhne, Orden unter dem Totenkopf, S. 427. Nach Müller-Hillebrand wies die Waffen-SS am Kriegsende noch eine Gesamtstärke von 830.000 Mann auf. Dazu muss man aber noch die bis dahin Gefallen zählen, deren Zahl nach Overmans Schätzung bei 314.000 Mann lag. Müller-Hillebrand geht insgesamt sogar von rund 1,15 Mio. Mann aus, die in der Waffen-SS gedient hätten. 
macht und Waffen-SS untersuchte, weist darauf hin, dass die Rekrutierung von Muslimen für die Waffen-SS zwar erst 1943 einsetzte, dass aber schon gut ein Jahr vorher muslimische Männer aus Osteuropa für Formationen der Wehrmacht geworben wurden. ${ }^{51}$ Für die Region Südosteuropa sind aber bereits Ende 1942 erste Rekrutierungen unter dem Kommando der Waffen-SS nachgewiesen, so beispielsweise die „Albanisch-Muselmanische Freiwilligenlegion“ im Raum Sandžak. ${ }^{52}$ Am 25. September 1943 berichtete der bosnische Verbindungsmann Nedim Salihbegović, dass bei früheren Rekrutierungen von „kroatischen Freiwilligen für die Ostfront“ der größte Anteil der Rekruten Muslime gewesen seien. ${ }^{53}$

Neben ideologischen Problemen barg die Rekrutierung „Fremdvölkischer“ aber auch zahlreiche praktische Schwierigkeiten: In welcher Sprache sollte exerziert, in welcher Sprache die Kommandos ausgegeben werden? ${ }^{54}$ Welche kulturellen Hintergründe, welche Ess- und Trinkgewohnheiten, welche religiösen Bräuche und Feiertage brachten diese Rekruten mit und wie sollte damit umgegangen werden? In welchem Maße, wann, wo und durch wen hatte die „ideologische“ bzw. „weltanschauliche Schulung“ und die militärische Ausbildung zu erfolgen? Neben der Bereitstellung von Schulungmaterialen für „fremdvölkische“ Verbände durch das SS-Hauptamt legte die SS-Führung besonderen Wert auf die Betonung von Gemeinsamkeiten zur "nordischen Rasse". 55 Die Frage nach der geistlichen Betreuung muslimischer Rekruten, aber auch nach der Art, wie mit ihnen im Alltag umzugehen sei, füllt ganze Akten. Aufrgund all dieser Fragen bildeten muslimische Soldaten innerhalb der „fremdvölkischen“ Soldaten der Waffen-SS eine eigene Kategorie.

51 Auf Initiative des Auswärtigen Amtes Ausland/Abwehr II wurden beispielsweise das „Turkbataillon 450“ und die „Deutsch-arabische Lehrabteilung“ aufgestellt. Zur selben Zeit stellte der SD krimtatarische „Selbstschutz“- und „Schutzmannschaftskompanien“ auf. Seit Frühjahr 1942 wurden die zu großen Teilen aus muslimischen „Turkvölkern“, aus Turkmenen, Turkestanern, Aserbeidschanern, Tschetschenen und Dagestanern und zu einem kleineren Teil aus christlichen Georgiern und Armeniern bestehenden „Ostlegionen“ aufgebaut, die schließlich rund 53.000 Mann, verteilt auf 53 Infanteriebataillone, zählen sollten. Vgl. Petke, Muslimische Soldaten, S. 249. Birn nennt bereits im Sommer und Herbst 1941 erste Aushebungen für sogenannte „Schutzmannschaften“ der Ordnungspolizei in den neu besetzten Ostgebieten, die als „unersetzliche Basis für den deutschen Repressionsapparat“ galten. Birn, Fremdvölkische, S. 61f. Vgl. auch Joachim Hoffmann, Die Ostlegionen 1941-1943, Turkotataren, Kaukasier und Wolgafinnen im deutschen Heer. Freiburg i.Br.: Rombach\&Co. 1981.

52 Vgl. Franziska Zaugg, Albanische Muslime, S. 180-184.

53 Vgl. Nedim Salihbegović, „Bericht zur Lage“, 25.9.1943, BArchB, NS 19/2601, Bl. 30.

54 Birn stellt bezüglich sprachlicher Schwierigkeiten bei fremdvölkischen Soldaten die Frage, ob sie „tatsächlich noch der Stärkung der deutschen Kampfkraft“ dienten. Birn, Fremdvölkische, S. 61.

55 Vgl. Birn, Fremdvölkische, S. 64 f. 